\title{
NEGATIVE DIFFERENTIAL RESISTANCE BEHAVIOR IN DELTA-DOPED AIInP STRUCTURE GROWN BY MOCVD
}

\author{
K. F. YARN* \\ Far East College, Department of Electrical Engineering, Optoelectronic Semiconductor Center, \\ Hsin-Shih Tainan, Taiwan 744, Republic of China
}

(Received 17 December 2001; In final form 21 March 2002)

\begin{abstract}
An AlInP delta-doped schottky diode exhibiting negative differential resistance (NDR) behavior is demonstrated for the first time. The NDR characteristics with a peak to valley ratio of 5.5 and peak current density of $1 \mathrm{kA} / \mathrm{cm}^{2}$ were achieved at room temperature. In addition, the maximum available power is estimated up to $5 \mathrm{~W} / \mathrm{cm}^{2}$. The mechanism for such performance is phenomenologically analyzed by the combination of resonant interband tunneling (RIT) and thermionic emission processes associated with tunneling effect on the metal-semiconductor (MS) interface.
\end{abstract}

Keywords: Delta-doped; Resonant interband tunneling (RIT)

\section{INTRODUCTION}

Delta doping technique shows the promising potential for introducing triangular barrier in semiconductor and attracts much attention to integrated circuits and high performance devices applications [1-3]. Recently, Wang et al. has reported that a symmetrical NDR characteristic with peak to valley current ratio (PVR) of 3.1 and peak current density of $3 \mathrm{kA} / \mathrm{cm}^{2}$ can be obtained in a homotype GaAs delta-doped structure operated at room temperature $[4,5]$. The peak current is resulted from the resonant interband tunneling process, while the valley current is dominated by the thermionic process. Based on this idea, a large band gap material is necessary to suppress the valley current, in order to obtain devices with high PVRs. Because AlInP material has a wider energy gap [6] than that of GaAs, it can be expected to reduce the valley current efficiently and then enhance the PVR.

In this paper, we demonstrate an AlInP delta-doped structure which shows N-shape NDR behavior with a high PVR up to 5.5 at room temperature. Due to the idea of using MS tunneling barrier, a conceptal understanding of such results would enhance our understanding of the physics of metal-AlInP RIT device in general.

\footnotetext{
* Present address: P.O. Box 345, Tainan, Taiwan 704, Republic of China.
} 


\section{EXPERIMENTAL}

AlInP delta-doped RIT structure in this study was grown on a (100)-oriented $p^{+}$-GaAs substrate by AIXTRON-2400 Metal Organic Chemical Vapor Deposition (MOCVD) system. TMGa, TMIn, TMAl and $\mathrm{AsH}_{3}, \mathrm{PH}_{3}$ are used as $\mathrm{MO}$ and hydride sources, respectively. $\mathrm{Si}_{2} \mathrm{H}_{6}$ is also used as n-type dopant source. This RIT structure consists of one Si deltadoped layer sandwitched between two lattice-matched undoped $\mathrm{Al}_{x} \mathrm{In}_{1-x} \mathrm{P}$ layer $(10 \mathrm{~nm})$ where $x$ is equal to 0.51 . The delta-doped sheet concentration is up to $1 \times 10^{12} \mathrm{~cm}^{-2}$. The corresponding energy band diagram is shown in Figure 1(a). Wafers are cleaned in acetone

(a)

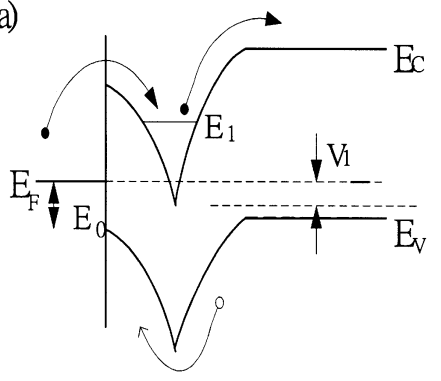

(b)

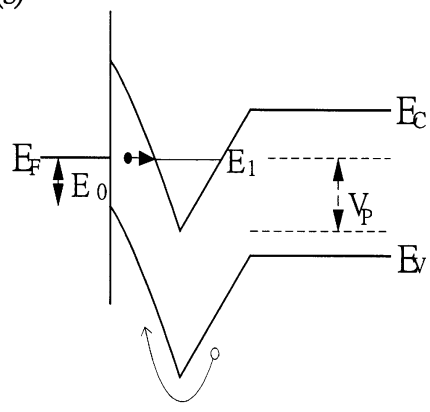

(c)

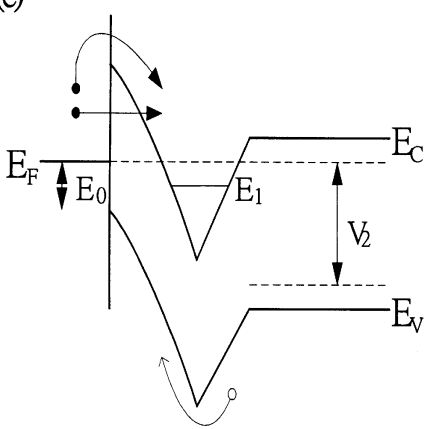

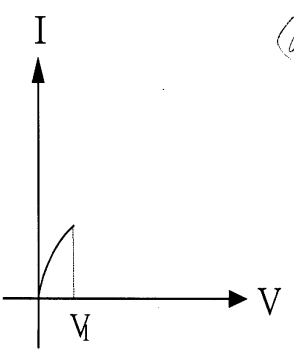
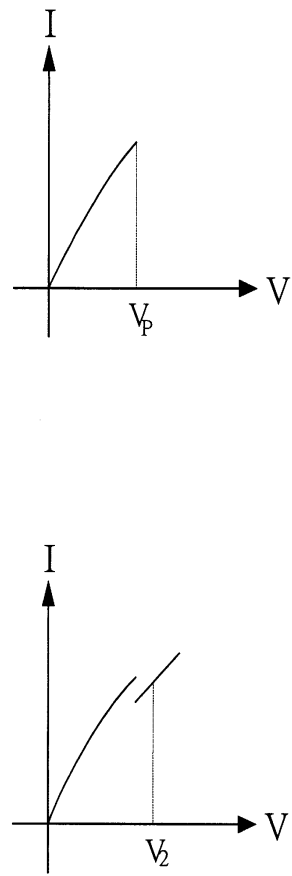

FIGURE 1 The energy band diagrams and current-voltage characteristics of AlInP delta-doped structure for various bias conditions: (a) small forward bias; (b) resonant tunneling condition; (c) large forward bias. 
and methanol for 5 minutes, respectively. Au is employed and evaporated on the surface of grown epitaxial layers as schottky metal. The device area of $1 \times 10^{-6} \mathrm{~cm}^{2}$ is defined by photolithography and mesa isolation is performed by using $\mathrm{H}_{2} \mathrm{SO}_{4}: 8 \mathrm{H}_{2} \mathrm{O}: 100 \mathrm{H}_{2} \mathrm{O}$ etching solution. Finally, the current-voltage $(I-V)$ characteristics are measured by a Tektronix $370 \mathrm{~A}$ curve tracer at room temperature.

\section{RESULTS AND DISCUSSION}

The room temperature $I-V$ characteristics of this RIT structure are shown in Figure 2, where an N-shaped NDR behavior is exhibited at forward bias. The corresponding peak voltage, peak current, valley voltage and valley current are $0.26 \mathrm{~V}, 1 \mathrm{~mA}, 0.4 \mathrm{~V}$ and $0.18 \mathrm{~mA}$, respectively. The calculated PVR is about 5.5 with a peak current density of $1 \mathrm{kA} / \mathrm{cm}^{2}$. This PVR value is higher than that of previously reported in homotype GaAs RIT diode [5]. Such an interesting NDR behavior is believed to be due to both surface state pinning effect and the tunneling effect through the delta-doped induced triangular quantum well. Thus, it means the validity of this NDR requires that the conduction band well created between the deltadoped barrier, is deep enough to allow the existence of quasi-bound states. In this case, NDR can occur via an interband tunneling mechanism. More detailed analyses are described as follows:

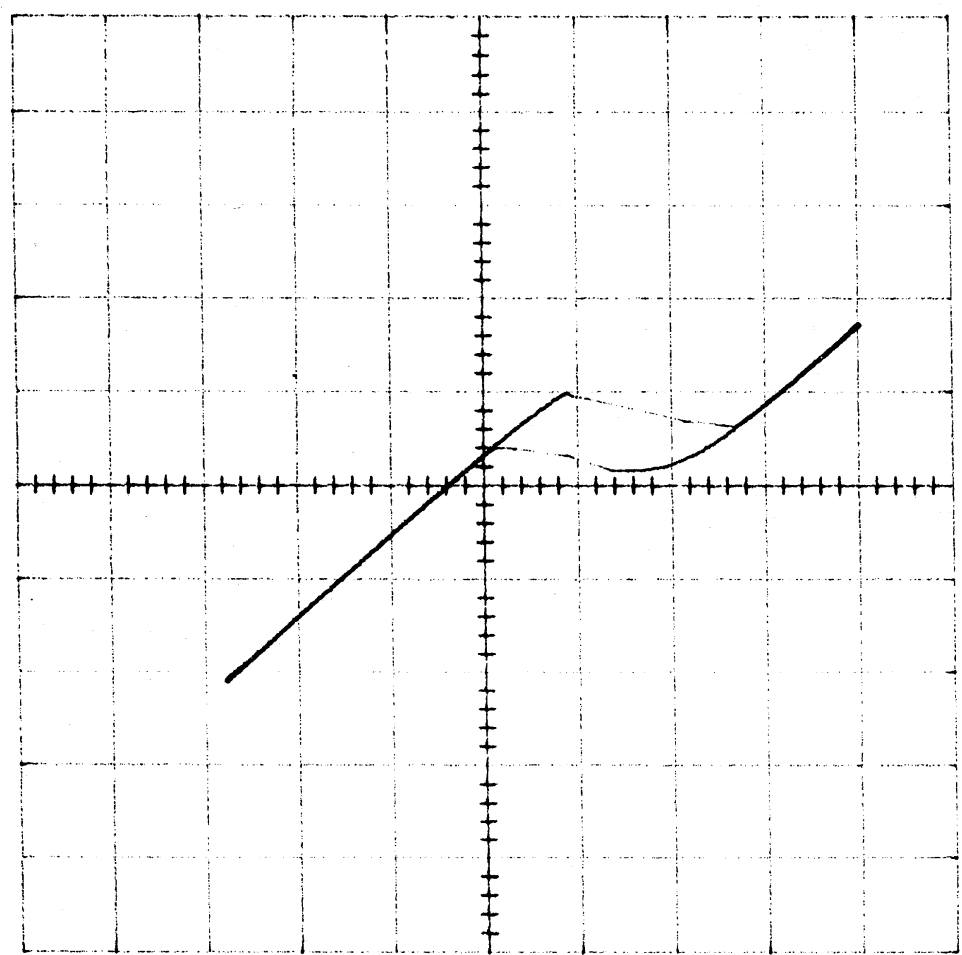

TYPE 37OA
VERTIDIV

$1 \mathrm{~mA}$

CURSOR

HORIZ/DIV

$200 \mathrm{mV}$

CURSOR

PER STEP

$100 n A$

DFFSET

OnA

$B$ or $9 \mathrm{~m} / D T V$

$10 K$

$\because$ OF COLLECTOR

PEAK VOLTS

12. 8

AUX SUPPLY

$0.00 \mathrm{~V}$

FIGURE 2 Typical current-voltage characteristic of the AlInP delta-doped structure at room temperature. 
At thermal equilibrium, the energy level of MS interface is pinned at $E_{0}$ which is located at the AlInP valence band. Since the delta-doped layer forms a triangular barrier, a quantized state $\left(E_{1}\right)$ exists in the conduction band region due to quantum size effect. In this condition, the confined state in triangular well lies above the Fermi level. When a small forward bias $\left(V_{1}\right)$ is applied, the electrons and holes will thermionic emission over the MS interface and delta-doped barrier, respectively. In this case, the collected current is increased when increasing the external voltage. When increasing the applied voltage, the electrons will tunnel through the MS interface via the resonance with the sublevel $\left(E_{1}\right)$ in triangular quantum well and then thermionic out of the well to the $p^{+}$.When the Fermi level aligns with the $E_{1}$ as shown in Fig. 1(b), a resonant tunneling process occurs. Due to the enhancement of tunneling probability, a peak current at the peak voltage $\left(V_{p}\right)$ is then established. As the bias is increased, the valence band edge is lowered and misaligned with the $E_{1}$, which makes electrons tunneling probability become small. This also reduces the tunneling current, which results in the formation of valley current. In other words, there is a drop in the current since electrons must tunnel through the whole AlInP layers, giving rise to the observed NDR effects. After that, the current is increased with the bias $\left(V_{2}\right)$ and the collected current is dominated by thermionic emission of electrons and holes associated with the electrons tunneling process in MS interface.

From experimental results, the maximum available power for such a NDR device can be estimated as $[7,8]$

$$
P_{\max }=\left(\frac{3}{16}\right) \Delta V \Delta I
$$

Where $\Delta V(\Delta I)$ is the voltage (current) extent of the NDR. Thus, a large power density of $5 \mathrm{~W} / \mathrm{cm}^{2}$ can also be expected in this structure.

\section{SUMMARY}

The first report of the observation of NDR behavior with a high PVR ratio of 5.5 and peak current density of $1 \mathrm{kA} / \mathrm{cm}^{2}$ are obtained in a MOCVD-grown AlInP delta-doped structure operated at room temperature. The occurrence of NDR is analyzed to be due to both the RIT effect in the triangular barrier well and thermionic emission associated with tunneling in MS interface. The calculated maximum available power is about $5 \mathrm{~W} / \mathrm{cm}^{2}$. Due to the high PVR, peak current density and maximum available power, microwave potential for such a new RIT device may have potential for future applications.

\section{References}

[1] Schubert, E. F., Kuo, J. M., Kope, R., Lufman, H. S., Hopkins, L. C. and Saner, N. J. (1990). Appl. Phys. Lett., 67, 1969.

[2] Malik, R. J., Aucoin, T. R., Ross, R. L., Board, K., Wood, C. E. C. and Eastman, L. F. (1980). Electronics Lett., 16, 836.

[3] Yarn, K. F., Wang, Y. H. and Chang, C. Y. (1990). Philo. Mag. Lett., 61, 339.

[4] Houng, M. P., Wang, Y. H., Chen, H. H. and Pan, C. C. (1992). Solid-state Electron., 35, 67.

[5] Wang, Y. H., Houng, M. P., Chen, H. H. and Wei, H. C. (1991). Electronics Lett., 27, 1667.

[6] Casey, Jr. and Panish, M. B. (1978). Heterostructure Lasers.

[7] Sollner, T. C. L. G., Tennenwald, P. E., Peck, D. D. and Goodhue, W. D. (1984). Appl. Phys. Lett., $45,1319$.

[8] Trambarulo, R. (1961). International Solid-state Circuits Conference. Philadelphia, P18. 

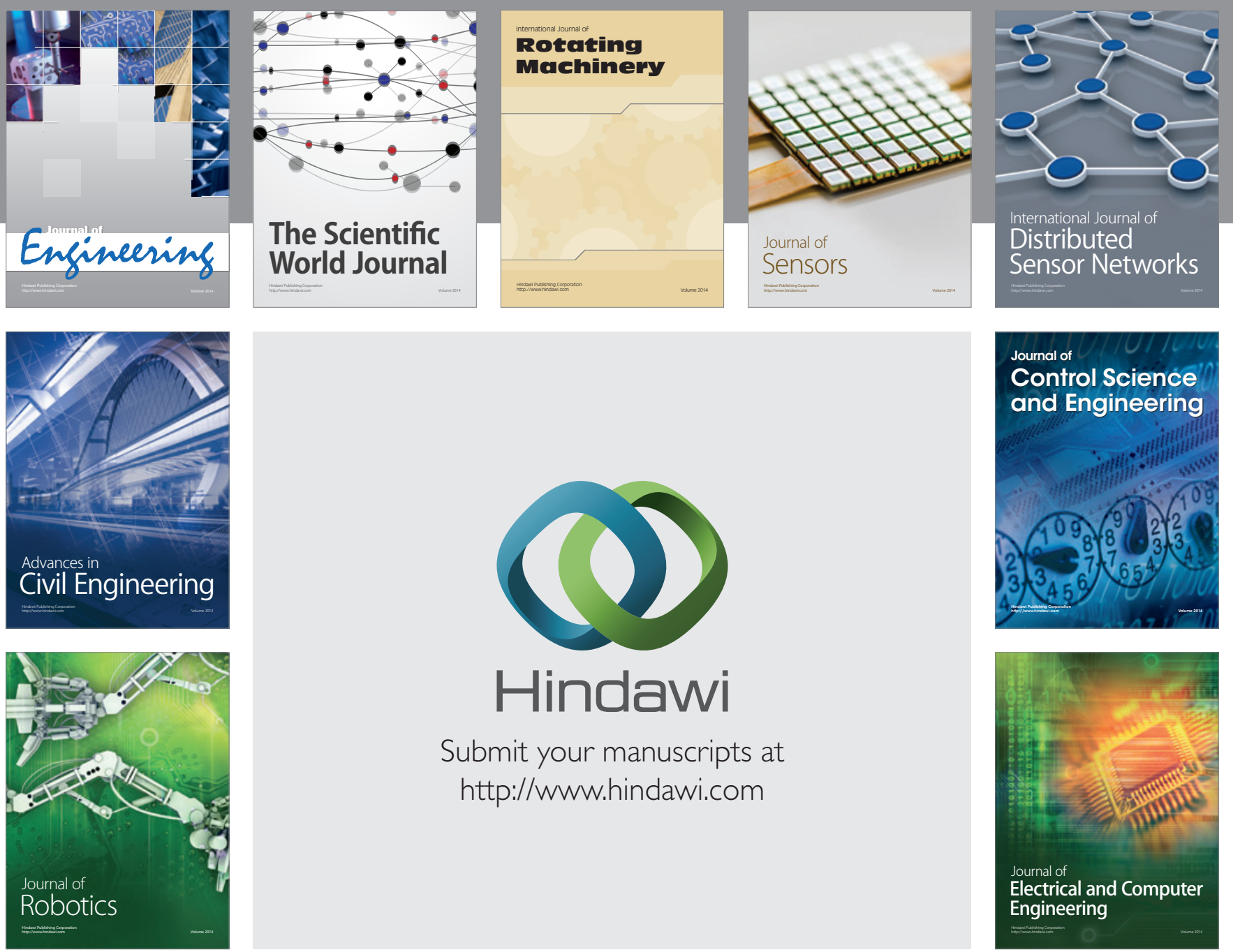

Submit your manuscripts at

http://www.hindawi.com
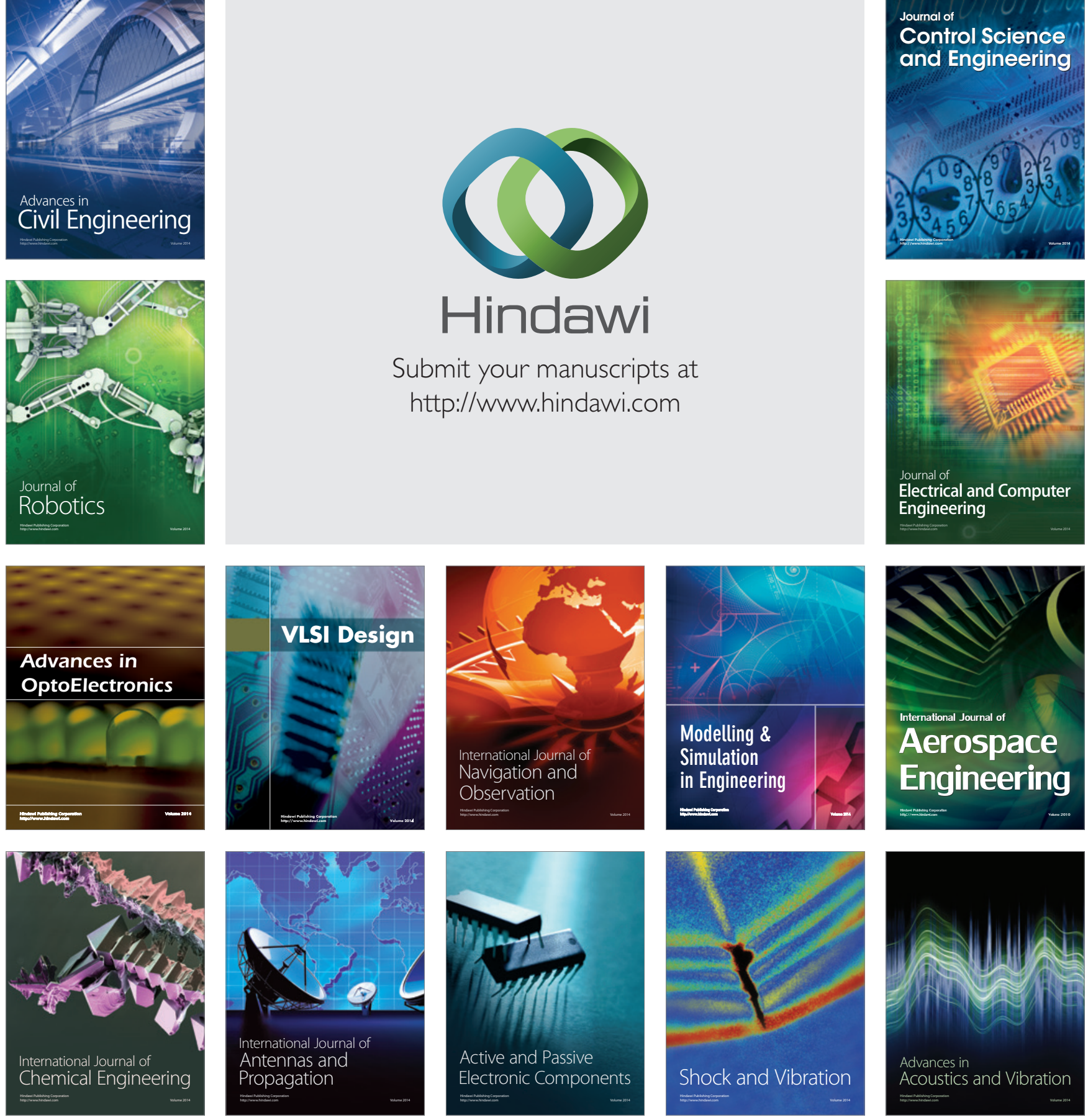